\title{
Guest editorial: design, modeling, sensing, actuation and control for micro/nanoscale systems
}

\author{
Hamid Reza Karimi ${ }^{1} \cdot$ Mingyang Xie ${ }^{2} \cdot$ Youmin Zhang $^{3}$ \\ Published online: 21 November 2019 \\ (C) Springer-Verlag London Ltd., part of Springer Nature 2019
}

Micro/nanoscale systems (MNSS) have been drawn much attention in control field recently due to its significance in various biomedical engineering applications. With the trend toward automation of the manipulations involved in biomedical engineering applications, developing an autonomous control MNSS is essential and urgently required. Furthermore, advanced design, modeling, sensing, actuation and control techniques are crucially important for the development of MNSS that can achieve biomedical processing with high speed, throughput, precision, and complexity. With the advances in micro-electro-mechanical systems (MEMS), robotics, and automatic control, it is opportunistic to exploit them for the benefit of the achievement of MNSS with high precision, complexity, and reliability.

Main intention of this special issue is to provide a premier international platform for wide range of professions including scholars, researchers, and engineers to share the latest advances in design, manufacturing, sensing, actuation, modeling, and control of MNSS. With a stringent peer review process, there are 9 papers accepted by this special issue, which are covering the following aspects: design for MNSS,

Hamid Reza Karimi

hamidreza.karimi@polimi.it

Mingyang Xie

myxie@nuaa.edu.cn

Youmin Zhang

ymzhang@encs.concordia.ca

1 Department of Mechanical Engineering, Politecnico di Milano, Milan, Italy

2 College of Automation Engineering, Nanjing University of Aeronautics and Astronautics, Nanjing, China

3 Department of Mechanical, Industrial, and Aerospace Engineering, Concordia University, Montreal, Canada modeling and control of MNSS, as well as biomedical applications. A summary of the accepted papers is discussed as follows:

\section{Design of MNSS}

Design of MNSS plays a cornerstone role in MNSS. Various platforms have been developed for the achievements of micro/ nanomanipulation over the past few decades, including MEMS, dielectrophoresis (DEP), magnetic field driven methods, optical field methods, hydrodynamic methods, and acoustic waves, etc. In the paper entitled "Design and control of a piezo-actuated microfeed mechanism for cell injection", the authors developed a novel microfeed mechanism for cell injection driven by a piezoelectric actuator (PEA) fulfill the requirement of a precise cell membrane puncture.

In the work named "Analysis of red blood cell deformability using parallel ladder electrodes in a microfluidic manipulation system" introduced a new method for the mechanical characterization of red blood cells (RBCs) on the basis of a dielectrophoresis (DEP) microfluidic system integrated with newly designed parallel ladder electrodes.

Another work entitled "Microscopic characterization and modeling of oxide layer for electrolytic in-process dressing (ELID) grinding with focus on voltage, electrode-wheel gap, and coolant flow" developed a system to observe how the oxide layer forms on the grinding surface during pre-dressing, and how the voltage, the gap between electrode and grinding wheel, and coolant flow rate affect the oxide layer thickness.

DEP has become a potential, robust, and flexible technique for cellular characterization, manipulation, separation, and cell patterning. Similar work can be found in "Cell patterning via optimized dielectrophoretic force within hexagonal electrodes in vitro for skin tissue engineering". Different from the previous work, in this paper, a thin-layer structure consisting of multiple hexagons for the regeneration of skin tissue was 
proposed. Cells could be seeded and cultured within the structure via dielectrophoresis (DEP) in three dimensions actively.

\section{Modeling and control of MNSS}

With ongoing development efforts towards automation of cell manipulation tasks, and the need to increase biological cell processing speed and throughput, an automated framework is required for the high accuracy and robustness control of biological cells. Modeling and control of MNSS are the two vital important techniques involved in the autonomous MNSS. The authors in the paper entitled "An improved algorithm based on $\sqrt{3}$ subdivision for micro surface modeling" proposes an improved uniform micro subdivision algorithm, based on $\sqrt{3}$ subdivision schemes, for micro surface modeling.

In the work named "A method to enhance fidelity of force feedback control in virtual and human-robot micro interaction cardiovascular intervention surgery" develops a dynamic model of the guide wire, which is used to simulate the collision of guide wire in the blood vessel. Furthermore, the terminal sliding mode controller is designed to improve the robustness and convergence of the force feedback control.

The authors in the paper with the title "Robust cooperative control for micro/nano scale systems subject to time-varying delay and structured uncertainties" presents a robust cooperative control problem of micro/nanoscale systems with timevarying delay and structured uncertainties.

The reported strategies for modeling and control of MNSS inspired by the approach adopted industrial applications. The authors in the paper with the title "Adaptive homographybased visual servo for micro unmanned surface vehicles" presented a novel adaptive homography-based visual servo (AHBVS) scheme to regulate a micro unmanned surface vehicle (MUSV) to the desired pose in the presence of both unknown image depth and unmatched dynamics. Another work entitled "Initial rotor position estimation of SPMSM based on voltage vector injection method" introduced an estimation method of initial rotor position of surface mounted permanent magnet synchronous motor (SPMSM) based on voltage vector injection.

Acknowledgments We really appreciate all the authors and anonymous reviewers who contributed to this special issue. Meanwhile, we would like to thank the supports from the Editor-in-Chief and Journal Editorial Staff to our special issue.

Publisher's note Springer Nature remains neutral with regard to jurisdictional claims in published maps and institutional affiliations. 